\title{
Necessary and sufficient conditions for oscillation of fourth order dynamic equations on time scales
}

\author{
Yong Zhou ${ }^{1,2^{*}}$ (D) Jia Wei He ${ }^{1}$, Bashir Ahmad ${ }^{2}$ and Ahmed Alsaedi²
}

\section{"Correspondence:}

yzhou@xtu.edu.cn

${ }^{1}$ Faculty of Mathematics and Computational Science, Xiangtan University, Hunan, P.R. China ${ }^{2}$ Nonlinear Analysis and Applied Mathematics (NAAM) Research Group, Faculty of Science, King Abdulaziz University, Jeddah, Saudi Arabia

\begin{abstract}
In this paper, we obtain necessary and sufficient conditions for oscillation of a fourth order dynamic equation on time scales with deviating arguments. We discuss the oscillation behavior of solutions for strongly superlinear and strongly sublinear cases of the dynamic equation at hand. Our results unify and improve some known results for dynamic equations on time scales.
\end{abstract}

MSC: 34C10; 34N05

Keywords: Dynamic equations; Time scales; Oscillation; Necessary and sufficient conditions

\section{Introduction}

The topic of oscillation and stability of dynamic equations on time scales has been developed very rapidly in the past two decades. There are some excellent monographs [1-4] and papers [5-10] containing some interesting works in the field.

The oscillatory behavior of solutions for nonlinear fourth order functional differential equations of the form

$$
\left[r(t) y^{\prime \prime}(t)\right]^{\prime \prime}+g(t, y(\eta(t)))=0, \quad t \geq t_{0}
$$

has been discussed by Onose [11], where $g$ is superlinear (sublinear) and strongly superlinear (strongly sublinear); he has extended and improved some interesting results of Kusano and Naito [12]. Furthermore, Gopalsamy et al. [13] obtained the sufficient and necessary conditions for oscillation of a fourth order differential equation with multiple deviating arguments given by

$$
\left[r(t) y^{\prime \prime}(t)\right]^{\prime \prime}+g\left(t, y\left(\eta_{1}(t)\right), y\left(\eta_{2}(t)\right), \ldots, y\left(\eta_{n}(t)\right)\right)=0, \quad t \geq t_{0},
$$

where $g$ is strongly superlinear and strongly sublinear.

For some more results on oscillation of solutions for different kinds of fourth order equations on time scales, see [14-21] and the references cited therein. However, it has been observed that there is no work in the related literature concerning the sufficient and nec-

(c) The Author(s) 2019. This article is distributed under the terms of the Creative Commons Attribution 4.0 International License (http://creativecommons.org/licenses/by/4.0/), which permits unrestricted use, distribution, and reproduction in any medium, provided you give appropriate credit to the original author(s) and the source, provide a link to the Creative Commons license, and indicate if changes were made. 
essary conditions for oscillation of fourth order dynamic equations on time scales. Motivated by the aforementioned works, in this paper, we consider the following fourth order dynamic equation with deviating arguments:

$$
\left[r(t) y^{\Delta \Delta}(t)\right]^{\Delta \Delta}+g(t, y(\eta(t)))=0, \quad t \in\left[t_{0}, \infty\right)=\mathbb{T}_{0} \subseteq \mathbb{T}
$$

where $y^{\Delta}(t)$ is the delta (or Hilger) derivative of $y$ at $t, r \in C_{r d}\left(\mathbb{T}_{0}, \mathbb{R}^{+}\right), \eta \in C_{r d}\left(\mathbb{T}_{0}, \mathbb{T}\right)$, $g: \mathbb{T}_{0} \times \mathbb{R} \rightarrow \mathbb{R}$ is a nonlinear continuous function, and $\operatorname{sgn} g(t, y)=\operatorname{sgn} y$ for $t \in \mathbb{T}_{0}$. In relation to (1), it is also assumed that $\int_{t_{0}}^{\infty} t / r(t) \Delta t=\infty$.

The paper is organized as follows. In Sect. 2, we recall some basic concepts of dynamic equations on time scales. In Sect. 3, we establish necessary and sufficient criteria for oscillation of (1) when $g$ is strongly superlinear as well as strongly sublinear.

\section{Preliminaries}

A time scale $\mathbb{T}$ is a nonempty closed subset of the real numbers $\mathbb{R}$ with $\sup \mathbb{T}=\infty$. For example, $\mathbb{R}, h \mathbb{Z}$ for $h>0$ and $q^{\mathbb{N}}:=\left\{q^{k}, k \in \mathbb{N}\right\}$ for $q>1$ are time scales. In the forthcoming analysis, we assume that $\mathbb{T}$ has the topology that it inherits from the standard topology on $\mathbb{R}$. Let the closed interval in $\mathbb{T}$ be defined by $[c, d]:=\{t \in \mathbb{T}, c \leq t \leq d\}$. In a similar manner, one can define open intervals and half-open intervals, etc.

Definition 2.1 For $t \in \mathbb{T}$ we define the forward jump operator $\sigma: \mathbb{T} \rightarrow \mathbb{T}$ by $\sigma(t):=\inf \{s \in$ $\mathbb{T}: s>t\}$; the backward jump operator $\rho: \mathbb{T} \rightarrow \mathbb{T}$ is defined by $\rho(t):=\sup \{s \in \mathbb{T}: s<t\}$. If $\sigma(t)>t$, then $t$ is called right-scattered, while if $\rho(t)<t$, it is called left-scattered. Also, if $t<\sup \mathbb{T}$ and $\sigma(t)=t$, then $t$ is called right-dense, and if $t>\inf \mathbb{T}$ and $\rho(t)=t$, then $t$ is called left-dense. The graininess function $\mu(t): \mathbb{T} \rightarrow[0, \infty)$ is defined by $\mu(t):=\sigma(t)-t$.

Definition 2.2 A function $f: \mathbb{T} \rightarrow \mathbb{R}$ is rd-continuous if it is continuous at all right-dense points and its left-sided limit exists (and is finite) at a left-dense point. We denote the set of rd-continuous functions by $C_{r d}(\mathbb{T}, \mathbb{R})$.

Definition 2.3 For a function $f: \mathbb{T} \rightarrow \mathbb{R}$, let $F^{\Delta}(t)$ represent the Hilger derivative of $f$ at $t$. Assume that $t_{0} \in \mathbb{T}$ and $f \in C_{r d}\left(\mathbb{T}_{0}, \mathbb{R}\right)$. If $F^{\Delta}(t)=f(t)$, then we define

$$
\int_{t_{0}}^{A} f(s) \Delta s:=F(A)-F\left(t_{0}\right), \quad \int_{t_{0}}^{\infty} f(s) \Delta s:=\lim _{A \rightarrow \infty} \int_{t_{0}}^{A} f(s) \Delta s
$$

Definition 2.4 We say $g$ is strongly superlinear if there exists a constant $\alpha>1$ such that

$$
\frac{|g(t, u)|}{|u|^{\alpha}} \leq \frac{|g(t, v)|}{|v|^{\alpha}} \text { for }|u| \leq|v|, u v>0, t \in \mathbb{T}_{0}
$$

while $g$ is strongly sublinear if there exists a constant $\alpha \in(0,1)$ such that

$$
\frac{|g(t, u)|}{|u|^{\alpha}} \geq \frac{|g(t, v)|}{|v|^{\alpha}} \text { for }|u| \leq|v|, u v>0, t \in \mathbb{T}_{0}
$$




\section{Main results}

In the sequel, we use the following notations:

$$
\begin{aligned}
& R(t)=\int_{t_{0}}^{t} \int_{t_{0}}^{s_{1}} \frac{s}{r(s)} \Delta s \Delta s_{1}, \quad R_{T}(t)=\int_{T}^{t} \int_{T}^{s_{1}} \frac{s-T}{r(s)} \Delta s \Delta s_{1}, \\
& \widetilde{\mathbf{R}}(t)=\int_{t_{0}}^{t} \frac{(t-s)\left(\sigma(s)-t_{0}\right)}{r(s)} \Delta s, \quad \widetilde{\mathbf{R}}_{u}(t)=\int_{u}^{t} \frac{(t-s)(\sigma(s)-u)}{r(s)} \Delta s, t>u>t_{0} .
\end{aligned}
$$

Lemma 3.1 If $y(t)$ is a nonoscillatory solution of (1), then there are only four cases for all sufficiently large $t \geq t_{0}$ :

(i) $\quad y(t)>0, \quad y^{\Delta}(t)>0, \quad r(t) y^{\Delta \Delta}(t)>0, \quad\left[r(t) y^{\Delta \Delta}\right]^{\Delta}>0$;

(ii) $\quad y(t)>0, \quad y^{\Delta}(t)>0, \quad r(t) y^{\Delta \Delta}(t)<0, \quad\left[r(t) y^{\Delta \Delta}\right]^{\Delta}>0$;

(iii) $\quad y(t)<0, \quad y^{\Delta}(t)<0, \quad r(t) y^{\Delta \Delta}(t)<0, \quad\left[r(t) y^{\Delta \Delta}\right]^{\Delta}<0$;

(iv) $\quad y(t)<0, \quad y^{\Delta}(t)<0, \quad r(t) y^{\Delta \Delta}(t)>0, \quad\left[r(t) y^{\Delta \Delta}\right]^{\Delta}<0$.

Proof Without loss of generality, let $y(t)$ be an eventually positive solution of (1), that is, there exists $t_{1} \geq t_{0}$ such that $y(t)>0$ for $t \geq t_{1}$. Then $y(\eta(t))>0$ for $t \geq t_{1}$. From (1), it yields that $\left[r(t) y^{\Delta \Delta}(t)\right]^{\Delta \Delta}<0$ for $t \geq t_{1}$. Therefore, $\left[r(t) y^{\Delta \Delta}(t)\right]^{\Delta}$ is eventually of constant sign. Next we suppose that $\left[r(t) y^{\Delta \Delta}(t)\right]^{\Delta}<0$ at some $t=t_{2} \geq t_{1}$. Then, integrating $\left[r(t) y^{\Delta \Delta}(t)\right]^{\Delta \Delta}<0$ twice from $t_{2}$ to $t$, and multiplying the resulting inequality by $1 / r(t)$ and integrating again from $t_{2}$ to $t$, we get

$$
y^{\Delta}(t)<\bar{a} \int_{t_{2}}^{t} \frac{s-t_{2}}{r(s)} \Delta s+\bar{b} \int_{t_{2}}^{t} \frac{1}{r(s)} \Delta s+\bar{c}, \quad t \geq t_{2}
$$

where $\bar{a}=\left[r\left(t_{2}\right) y^{\Delta \Delta}\left(t_{2}\right)\right]^{\Delta}<0, \bar{b}=r\left(t_{2}\right) y^{\Delta \Delta}\left(t_{2}\right)$, and $\bar{c}=y^{\Delta}\left(t_{2}\right)$. In consequence, it follows from the assumption $\int_{t_{0}}^{\infty} s / r(s) \Delta s=\infty$ that $\lim _{t \rightarrow \infty} y^{\Delta}(t)=-\infty$, which contradicts the positivity of $y(t)$. Therefore, we have $\left[r(t) y^{\Delta \Delta}(t)\right]^{\Delta}>0$ for all $t \geq t_{1}$. It means that $r(t) y^{\Delta \Delta}(t)$ eventually keeps the same sign. On the other hand, let $r(t) y^{\Delta \Delta}(t)<0$ for $t \geq t_{1}$. Then it can easily be shown that $y^{\Delta}(t)$ is eventually positive. This completes the proof of (i). If there exists $t_{2} \geq t_{1}$ such that $r(t) y^{\Delta \Delta}(t)>0$ for $t \geq t_{2}$, then $r(t) y^{\Delta \Delta}(t) \geq c$ for $t \geq t_{2}$, where $c=r\left(t_{2}\right) y^{\Delta}\left(t_{2}\right)$. Multiplying this inequality by $t / r(t)$ and integrating from $t_{2}$ to $t$, by using the integration by parts formula on time scales, we get

$$
t y^{\Delta}(t)-y(\sigma(t))-t_{2} y^{\Delta}\left(t_{2}\right)+y\left(\sigma\left(t_{2}\right)\right) \geq c \int_{t_{2}}^{t} \frac{s}{r(s)} \Delta s, \quad t \geq t_{2}
$$

which, together with $\int_{t_{0}}^{\infty} s / r(s) \Delta s=\infty$, implies that $\lim _{t \rightarrow \infty} t y^{\Delta}(t)=\infty$. Thus $y^{\Delta}(t)>0$ for all large $t \geq t_{0}$. The proof is completed.

Lemma 3.2 If $t_{1} \geq t_{0}$ and $t>u$, then $\lim _{t \rightarrow \infty} \frac{R_{t_{1}}(t)}{R(t)}=1$ and $\widetilde{\mathbf{R}}_{u}(t)$ is nonincreasing for $u$, where $R_{t_{1}}(t), R(t), \widetilde{\mathbf{R}}_{u}(t)$ are given in (2). 
Proof By applying L'Hôpital's rule [1, Theorem 1.120], we find that

$$
\lim _{t \rightarrow \infty} \frac{R_{t_{1}}(t)}{R(t)}=\lim _{t \rightarrow \infty} \frac{\int_{t_{1}}^{t} \int_{t_{1}}^{s_{1}} \frac{s-t_{1}}{r(s)} \Delta s \Delta s_{1}}{\int_{t_{0}}^{t} \int_{t_{0}}^{s_{1}} \frac{s}{r(s)} \Delta s \Delta s_{1}}=\lim _{t \rightarrow \infty} \frac{\int_{t_{1}}^{t} \frac{s-t_{1}}{r(s)} \Delta s}{\int_{t_{0}}^{t} \frac{s}{r(s)} \Delta s}=\lim _{t \rightarrow \infty} \frac{\frac{t-t_{1}}{r(t)}}{\frac{t}{r(t)}}=1 .
$$

On the other hand, let

$$
f(u, s)=\frac{(t-s)(\sigma(s)-u)}{r(s)}, \quad t_{0}<u<s<t .
$$

Then, using [1, Theorem 1.117] for all $u$, we obtain

$$
\left[\widetilde{\mathbf{R}}_{u}(t)\right]^{\Delta}=\int_{u}^{t}[f(u, s)]^{\Delta} \Delta s-f(\sigma(u), u)=-\int_{u}^{t} \frac{t-s}{r(s)} \Delta s \leq 0 .
$$

This completes the proof.

Lemma 3.3 If $y(t)$ is a nonoscillatory solution of (1), then there exist $T>t_{0}$ and a constant $\tilde{c}>0$ such that

$$
\frac{1}{2}\left[r(t) y^{\Delta \Delta}(t)\right]^{\Delta} R(t) \leq|y(t)| \leq \tilde{c} R(t) \quad \text { for } t \geq T .
$$

Proof Without loss of generality, we suppose that $y(t)$ is eventually positive. Then, in view of Lemma 3.1, there exists $t_{1} \geq t_{0}$ such that

$$
y(t)>0, \quad y^{\Delta}(t)>0, \quad\left[r(t) y^{\Delta \Delta}(t)\right]^{\Delta}>0, \quad\left[r(t) y^{\Delta \Delta}(t)\right]^{\Delta \Delta}<0 \quad \text { for } t \geq t_{1} .
$$

Integrating $\left[r(t) y^{\Delta \Delta}(t)\right]^{\Delta \Delta}<0$ twice from $t_{1}$ to $t$, we have

$$
r(t) y^{\Delta \Delta}(t)<a_{0} t+a_{1}, \quad \text { where } a_{0}=\left[r\left(t_{1}\right) y^{\Delta \Delta}\left(t_{1}\right)\right]^{\Delta}>0, a_{1}=r\left(t_{1}\right) y^{\Delta \Delta}\left(t_{1}\right) .
$$

Multiplying (4) by $1 / r(t)$ and integrating twice from $t_{1}$ to $t$ yields

$$
y(t)<a_{0} \int_{t_{1}}^{t} \int_{t_{1}}^{s_{1}} \frac{s}{r(s)} \Delta s \Delta s_{1}+a_{1} \int_{t_{1}}^{t} \int_{t_{1}}^{s_{1}} \frac{1}{r(s)} \Delta s \Delta s_{1}+a_{2} t+a_{3},
$$

where $a_{2}=y^{\Delta}\left(t_{1}\right)$ and $a_{3}=y\left(t_{1}\right)$ are constants. Noting that $\int_{t_{0}}^{\infty} s / r(s) \Delta s=\infty$, we deduce that there exist $t_{2} \geq t_{1}$ and $\tilde{c}>0$ such that $y(t)<\tilde{c} R(t)$ for $t \geq t_{2}$.

Now let us prove the left-sided inequality in the lemma. In view of (3), observe that $\left[r(t) y^{\Delta \Delta}(t)\right]^{\Delta}$ is nonincreasing, and hence

$$
\begin{aligned}
y(t) & \geq \int_{t_{1}}^{t} y^{\Delta}(s) \Delta s \geq \int_{t_{1}}^{t} \int_{t_{1}}^{s_{1}} y^{\Delta \Delta}(s) \Delta s \Delta s_{1}=\int_{t_{1}}^{t} \int_{t_{1}}^{s_{1}} \frac{1}{r(s)} r(s) y^{\Delta \Delta}(s) \Delta s \Delta s_{1} \\
& \geq \int_{t_{1}}^{t} \int_{t_{1}}^{s_{1}} \frac{1}{r(s)} \int_{t_{1}}^{s}\left[r(u) y^{\Delta \Delta}(u)\right]^{\Delta} \Delta u \Delta s \Delta s_{1} \\
& \geq\left[r(t) y^{\Delta \Delta}(t)\right]^{\Delta} \int_{t_{1}}^{t} \int_{t_{1}}^{s_{1}} \frac{1}{r(s)} \int_{t_{1}}^{s} \Delta u \Delta s \Delta s_{1}
\end{aligned}
$$




$$
=\left[r(t) y^{\Delta \Delta}(t)\right]^{\Delta} \int_{t_{1}}^{t} \int_{t_{1}}^{s_{1}} \frac{s-t_{1}}{r(s)} \Delta s \Delta s_{1}=\left[r(t) y^{\Delta \Delta}(t)\right]^{\Delta} R_{t_{1}}(t) .
$$

From Lemma 3.2, there exists $t_{3} \geq t_{1}$ such that $R_{t_{1}}(t) \geq \frac{1}{2} R(t)$ for $t \geq t_{3}$, and hence

$$
y(t) \geq \frac{1}{2}\left[r(t) y^{\Delta \Delta}(t)\right]^{\Delta} R(t) .
$$

Letting $T=\max \left(t_{2}, t_{3}\right)$, the proof is complete.

Lemma 3.4 If $y(t)$ is a nonoscillatory solution of (1), then there exists $t^{*} \geq t_{0}$ such that, for any $T \geq t^{*}$,

$$
y(t) \geq \int_{t_{1}}^{t} \widetilde{\mathbf{R}}_{t_{1}}(\sigma(s)) g(s, y(\eta(s))) \Delta s, \quad t \geq T .
$$

Proof Without loss of generality, we suppose that $y(t)$ is eventually positive. Firstly, if $y(t)$ is a solution of type-(i), then there exists $t_{1} \geq t_{0}$ such that

$$
y(t)>0, \quad y^{\Delta}(t)>0, \quad r(t) y^{\Delta \Delta}(t)>0, \quad\left[r(t) y^{\Delta \Delta}(t)\right]^{\Delta}>0 \quad \text { for } t \geq t_{1} .
$$

Let

$$
h(t)=y(t)-\int_{t_{1}}^{t} \int_{s_{3}}^{\infty} \frac{1}{r\left(s_{2}\right)} \int_{s_{2}}^{\infty} \int_{s_{1}}^{\infty} g(s, y(\eta(s))) \Delta s \Delta s_{1} \Delta s_{2} .
$$

Obviously, $h^{\Delta \Delta}(t)>0$ for $t \geq t_{1}$. Indeed, differentiating the above equation twice, we get

$$
h^{\Delta \Delta}(t)=y^{\Delta \Delta}(t)+\frac{1}{r(t)} \int_{t}^{\infty} \int_{s_{1}}^{\infty} g(s, y(\eta(s))) \Delta s \Delta s_{1} \Delta s_{2}>0 .
$$

In view of (1), we obtain $\left[r(t) h^{\Delta \Delta}(t)\right]^{\Delta \Delta}=0$ and hence $\left[r(t) h^{\Delta \Delta}(t)\right]^{\Delta}=c$. Integrating (1) from $t$ to $T$, we have

$$
\left[r(t) y^{\Delta \Delta}(t)\right]^{\Delta}-\int_{t}^{T} g(s, y(\eta(s))) \Delta s=\left[r(T) y^{\Delta \Delta}(T)\right]^{\Delta}>0 .
$$

In the limit $T \rightarrow \infty$, we note that $\left[r(t) h^{\Delta \Delta}(t)\right]^{\Delta}>0$ for $t \geq t_{1}$. Then there exists $c>0$ such that $h^{\Delta \Delta}(t)=c t / r(t)>0$ for $t \geq t_{1}$, which, on integrating from $t_{1}$ to $t$, yields

$$
h^{\Delta}(t)=h^{\Delta}\left(t_{1}\right)+c \int_{t_{1}}^{t} s / r(s) \Delta s .
$$

Taking the limit $t \rightarrow \infty$ and using the assumption $\int_{t_{0}}^{\infty} s / r(s) \Delta s=\infty$ in (6), we get $h^{\Delta}(t)>0$ for all large $t$. Therefore, there exists $t_{2} \geq t_{1}$ such that $h^{\Delta}(t)>c \int_{t_{1}}^{t} s / r(s) \Delta s$ for $t \geq t_{2}$. Next, integrating (6) from $t_{1}$ to $t$, we get

$$
h(t)=h\left(t_{1}\right)+c \int_{t_{1}}^{t} \int_{t_{1}}^{s_{1}} s / r(s) \Delta s \Delta s_{1}, \quad t \geq t_{2}
$$


which implies that $h(t)>0$ for large values of $t$ (i.e., $t \rightarrow \infty$ ). Thus, there exists $T \geq t_{2}$ such that

$$
y(t) \geq \int_{t_{1}}^{t} \int_{s_{3}}^{\infty} \frac{1}{r\left(s_{2}\right)} \int_{s_{2}}^{\infty} \int_{s_{1}}^{\infty} g(s, y(\eta(s))) \Delta s \Delta s_{1} \Delta s_{2} \Delta s_{3}, \quad t \geq T .
$$

Now, by interchanging the order of integration, we get

$$
\begin{aligned}
y(t) \geq & \int_{t_{1}}^{t} \int_{t_{1}}^{\sigma\left(s_{2}\right)} \frac{1}{r\left(s_{2}\right)} \int_{s_{2}}^{\infty}\left(\sigma(s)-s_{2}\right) g(s, y(\eta(s))) \Delta s \Delta s_{3} \Delta s_{2} \\
& +\int_{t}^{\infty} \int_{T}^{t} \frac{1}{r\left(s_{2}\right)} \int_{s_{2}}^{\infty}\left(\sigma(s)-s_{2}\right) g(s, y(\eta(s))) \Delta s \Delta s_{3} \Delta s_{2} \\
\geq & \int_{t_{1}}^{t} \frac{\sigma\left(s_{2}\right)-t_{1}}{r\left(s_{2}\right)} \int_{s_{2}}^{\infty}\left(\sigma(s)-s_{2}\right) g(s, y(\eta(s))) \Delta s \Delta s_{2} \\
= & \int_{t_{1}}^{t} \int_{t_{1}}^{\sigma(s)} \frac{\left(\sigma(s)-s_{2}\right)\left(\sigma\left(s_{2}\right)-t_{1}\right)}{r\left(s_{2}\right)} g(s, y(\eta(s))) \Delta s_{2} \Delta s \\
& +\int_{t}^{\infty} \int_{t_{1}}^{t} \frac{\left(\sigma(s)-s_{2}\right)\left(\sigma\left(s_{2}\right)-t_{1}\right)}{r\left(s_{2}\right)} g(s, y(\eta(s))) \Delta s_{2} \Delta s \\
\geq & \int_{t_{1}}^{t} \widetilde{\mathbf{R}}_{t_{1}}(\sigma(s)) g(s, y(\eta(s))) \Delta s .
\end{aligned}
$$

On the other hand, if $y(t)$ is a solution of type-(ii), then there exists $t_{3} \geq t_{0}$ such that

$$
y(t)>0, \quad y^{\Delta}(t)>0, \quad r(t) y^{\Delta \Delta}(t)<0, \quad\left[r(t) y^{\Delta \Delta}(t)\right]^{\Delta}>0 \quad \text { for } t \geq t_{3} .
$$

Integrating (1) from $t$ to $T$, we get

$$
\left[r(T) y^{\Delta \Delta}(T)\right]^{\Delta}-\left[r(t) y^{\Delta \Delta}(t)\right]^{\Delta}+\int_{t}^{T} g(s, y(\eta(s))) \Delta s=0,
$$

which takes the following form after taking the limit $T \rightarrow \infty$ :

$$
\left[r(t) y^{\Delta \Delta}(t)\right]^{\Delta} \geq \int_{t}^{\infty} g(s, y(\eta(s))) \Delta s, \quad t \geq t_{3} .
$$

Integrating the above inequality from $t$ to $T$, we have

$$
r(T) y^{\Delta \Delta}(T)-r(t) y^{\Delta \Delta}(t) \geq \int_{t}^{T} \int_{s_{1}}^{\infty} g(s, y(\eta(s))) \Delta s \Delta s_{1} .
$$

Multiplying the above inequality by $1 / r(t)$, and then integrating from $t$ to $T$, we get

$$
y^{\Delta}(t) \geq y^{\Delta}(T)+r(T) y^{\Delta \Delta}(T)(t-T)+\int_{t}^{T} \frac{1}{r\left(s_{2}\right)} \int_{s_{2}}^{T} \int_{s_{1}}^{\infty} g(s, y(\eta(s))) \Delta s \Delta s_{1} \Delta s_{2},
$$

which, on taking the limit $T \rightarrow \infty$, yields

$$
y^{\Delta}(t) \geq \int_{t}^{\infty} \frac{1}{r\left(s_{2}\right)} \int_{s_{2}}^{\infty} \int_{s_{1}}^{\infty} g(s, y(\eta(s))) \Delta s \Delta s_{1} \Delta s_{2}, \quad t \geq t_{3} .
$$


Integrating the above inequality from $t_{1}$ to $t$, we get

$$
y(t) \geq \int_{t_{1}}^{t} \int_{s_{3}}^{\infty} \frac{1}{r\left(s_{2}\right)} \int_{s_{2}}^{\infty} \int_{s_{1}}^{\infty} g(s, y(\eta(s))) \Delta s \Delta s_{1} \Delta s_{2}, \quad t \geq t_{3}
$$

Using the argument employed in (7) and defining $t^{*}=\max \left(t_{2}, t_{3}\right)$, we deduce that the conclusion of the lemma holds.

Lemma 3.5 Let $f$ and $g$ be $\Delta$-differentiable on $\mathbb{T}$. Assume that $g(t), g^{\Delta}(t)$ are not equal to zero for all $t \in \mathbb{T}$ and have the same sign. Then

$$
\lim _{t \rightarrow \infty} \frac{f(\sigma(t))}{g(\sigma(t))}=\xi
$$

if

$$
\lim _{t \rightarrow \infty} \frac{f(t)}{g(t)}=\lim _{t \rightarrow \infty} \frac{f^{\Delta}(t)}{g^{\Delta}(t)}=\xi \in \mathbb{R}
$$

Proof Since $g(t)$ and $g^{\Delta}(t)$ are not equal to zero for all $t \in \mathbb{T}$, it follows from the identity $g(\sigma(t))=\mu(t) g^{\Delta}(t)+g(t)$ that $g(\sigma(t))$ is not equal to zero for all $t \in \mathbb{T}$. Hence, for any $\varepsilon>0$, there exists $T>t_{0}$ such that, for $t \geq T$, we have

$$
\left|\frac{f(t)}{g(t)}-\xi\right| \leq \varepsilon \quad \text { and } \quad\left|\frac{f^{\Delta}(t)}{g^{\Delta}(t)}-\xi\right| \leq \varepsilon
$$

If $\operatorname{sgn} g(t)=\operatorname{sgn} g^{\Delta}(t)>0$, then

$$
\xi g(t)-\varepsilon g(t) \leq f(t) \leq \xi g(t)+\varepsilon g(t) \quad \text { and } \quad \xi g^{\Delta}(t)-\varepsilon g^{\Delta}(t) \leq f^{\Delta}(t) \leq \xi g^{\Delta}(t)+\varepsilon g^{\Delta}(t) .
$$

Hence, noticing that $f(\sigma(t))=\mu(t) f^{\Delta}(t)+f(t)$ and $g(\sigma(t))>0$, we get

$$
\xi g(\sigma(t))-\varepsilon g(\sigma(t)) \leq f(\sigma(t)) \leq \xi g(\sigma(t))+\varepsilon g(\sigma(t)), \quad t \geq T .
$$

Consequently, we have

$$
\left|\frac{f(\sigma(t))}{g(\sigma(t))}-\xi\right| \leq \varepsilon, \quad t \geq T
$$

Also one can observe that the above expression holds for $\operatorname{sgn} g(t)=\operatorname{sgn} g^{\Delta}(t)<0$. Thus, in view of the arbitrariness of $\varepsilon$, we obtain the desired result.

Theorem 3.6 Assume that $g$ is strongly superlinear and $\eta(t) \geq \sigma(t)$. Then every solution of (1) is oscillatory if and only if

$$
\int_{t_{0}}^{\infty} \widetilde{\mathbf{R}}(\sigma(t))|g(t, c)| \Delta t=\infty \quad \text { for all } c \neq 0
$$


Proof We first prove the necessity by contradiction. Let us suppose that condition (8) does not hold true. Then there exists a positive constant $c$ such that

$$
\int_{t_{0}}^{\infty} \widetilde{\mathbf{R}}(\sigma(t))|g(t, c)| \Delta t<\infty
$$

So we can choose a sufficiently large $T>t_{0}$ such that

$$
\int_{T}^{\infty} \widetilde{\mathbf{R}}(\sigma(t))|g(t, c)| \Delta t<\frac{c}{4}
$$

Let

$$
U=\left\{y\left|y \in C_{r d}\left(\mathbb{T}_{0}, \mathbb{R}\right), \sup _{t \in \mathbb{T}_{0}}\right| y(t) \mid<\infty\right\}
$$

It is clear that $U$ is a Banach space with the norm $\|y\|=\sup _{t \in \mathbb{T}_{0}}|y(t)|$. Let us introduce a closed, bounded, and convex subset of $U$ defined by

$$
\Omega=\left\{y=y(t): y \in U, \frac{c}{2} \leq|y(t)| \leq c, t \in \mathbb{T}_{0}\right\}
$$

Define a map $\mathcal{P}$ on $\Omega$ as follows:

$$
(\mathcal{P} y)(t)= \begin{cases}\frac{c}{2}+\int_{T}^{t} \int_{s_{3}}^{\infty} \frac{1}{r\left(s_{2}\right)} \int_{s_{2}}^{\infty} \int_{s_{1}}^{\infty} g(s, y(\eta(s))) \Delta s \Delta s_{1} \Delta s_{2} \Delta s_{3}, & t \geq T, \\ (\mathcal{P} y)(T), & t_{0} \leq t \leq T .\end{cases}
$$

In the sequel, we will show that $\mathcal{P}$ has a fixed point in $\Omega$.

Step I. $\mathcal{P}$ maps $\Omega$ into $\Omega$. Let $y \in \Omega$. Then $c / 2 \leq|y(t)| \leq c$ for $t \geq t_{0}$. In view of Lemma 3.2, we have that $\widetilde{\mathbf{R}}_{T}(t) \leq \widetilde{\mathbf{R}}(t)$ for $T \geq t_{0}$. Then, for $t \geq T$, we obtain

$$
\begin{aligned}
|(\mathcal{P} y)(t)| \leq & \frac{c}{2}+\int_{T}^{t} \int_{s_{3}}^{\infty} \frac{1}{r\left(s_{2}\right)} \int_{s_{2}}^{\infty} \int_{s_{1}}^{\infty}|g(s, y(\eta(s)))| \Delta s \Delta s_{1} \Delta s_{2} \Delta s_{3} \\
= & \frac{c}{2}+\int_{T}^{t} \int_{T}^{\sigma\left(s_{2}\right)} \frac{1}{r\left(s_{2}\right)} \int_{s_{2}}^{\infty}\left(\sigma(s)-s_{2}\right)|g(s, y(\eta(s)))| \Delta s \Delta s_{3} \Delta s_{2} \\
& +\int_{t}^{\infty} \int_{T}^{t} \frac{1}{r\left(s_{2}\right)} \int_{s_{2}}^{\infty}\left(\sigma(s)-s_{2}\right)|g(s, y(\eta(s)))| \Delta s \Delta s_{3} \Delta s_{2} \\
\leq & \frac{c}{2}+2 \int_{T}^{\infty} \frac{\sigma\left(s_{2}\right)-T}{r\left(s_{2}\right)} \int_{s_{2}}^{\infty}\left(\sigma(s)-s_{2}\right)|g(s, y(\eta(s)))| \Delta s \Delta s_{2} \\
= & \frac{c}{2}+2 \int_{T}^{\infty} \int_{T}^{\sigma(s)} \frac{\sigma\left(s_{2}\right)-T}{r\left(s_{2}\right)}\left(\sigma(s)-s_{2}\right) g(s, y(\eta(s))) \Delta s_{2} \Delta s \\
\leq & \frac{c}{2}+2 \int_{T}^{\infty} \widetilde{\mathbf{R}}(\sigma(s))|g(s, y(\eta(s)))| \Delta s .
\end{aligned}
$$

Then it follows from the strong superlinearity of $g$ that

$$
\frac{c}{2} \leq|(\mathcal{P} y)(t)| \leq \frac{c}{2}+2 \int_{T}^{\infty} \widetilde{\mathbf{R}}(\sigma(s))|g(s, y(\eta(s)))| \Delta s<\frac{c}{2}+2 \int_{T}^{\infty} \widetilde{\mathbf{R}}(\sigma(s))|g(s, c)| \Delta s \leq c,
$$

which implies that $c / 2 \leq(\mathcal{P} y)(t) \leq c$ for $t \in \mathbb{T}_{0}$. This shows that $\mathcal{P} \Omega \subseteq \Omega$. 
Step II. $\mathcal{P}$ is completely continuous.

We first show that $\mathcal{P}$ is continuous. Let $y_{n} \in \Omega(n=1,2, \ldots)$ such that $\left\|y_{n}-y\right\| \rightarrow 0$ as $n \rightarrow \infty$. Hence we get $y \in \Omega$ since $\Omega$ is a closed set. Then

$$
\begin{aligned}
& \left|\left(\mathcal{P} y_{n}\right)(t)-(\mathcal{P} y)(t)\right| \\
& \quad \leq \int_{T}^{t} \int_{s_{3}}^{\infty} \frac{1}{r\left(s_{2}\right)} \int_{s_{2}}^{\infty} \int_{s_{1}}^{\infty}\left|g\left(s, y_{n}(\eta(s))\right)-g(s, y(\eta(s)))\right| \Delta s \Delta s_{1} \Delta s_{2} \Delta s_{3} \\
& \quad \leq 2 \int_{T}^{\infty} \frac{\sigma\left(s_{2}\right)-T}{r\left(s_{2}\right)} \int_{s_{2}}^{\infty}\left(\sigma(s)-s_{2}\right)\left|g\left(s, y_{n}(\eta(s))\right)-g(s, y(\eta(s)))\right| \Delta s \Delta s_{2} \\
& \quad \leq 2 \int_{T}^{\infty} \widetilde{\mathbf{R}}(\sigma(s))\left|g\left(s, y_{n}(\eta(s))\right)-g(s, y(\eta(s)))\right| \Delta s,
\end{aligned}
$$

which, by the strong superlinearity of $g$, yields

$$
|g(s, y(\eta(s)))| \leq|g(s, c)|, \quad \text { and } \quad\left|g\left(s, y_{n}(\eta(s))\right)\right| \leq|g(s, c)|, \quad n=1,2 \ldots
$$

In consequence, we get

$$
\left|g\left(s, y_{n}(\eta(s))\right)-g(s, y(\eta(s)))\right| \leq 2|g(s, c)| \text {. }
$$

Since $\left|g\left(s, y_{n}(\eta(s))\right)-g(s, y(\eta(s)))\right| \rightarrow 0$ as $n \rightarrow \infty$, the Lebesgue dominated convergence theorem implies that $\lim _{n \rightarrow \infty}\left\|\mathcal{P} y_{n}-\mathcal{P} y\right\|=0$, and hence we obtain that $\mathcal{P}$ is continuous in $\Omega$.

Next, we show that $\mathcal{P} \Omega$ is relatively compact. According to the Arzela-Ascoli theorem on time scales (see [6]), we just need to verify that the family of functions $\{\mathcal{P} y: y \in \Omega\}$ is bounded and uniformly Cauchy, and $\{\mathcal{P} y: y \in \Omega\}$ is equi-continuous on $\left[t_{0}, T_{1}\right]$ for any $T_{1} \in \mathbb{T}_{0}$. Firstly, the boundedness is obvious. Secondly, in view of (9), for any $\varepsilon>0$, we can choose a sufficiently large number $T^{*} \geq T$ so that

$$
\int_{T^{*}}^{\infty} \widetilde{\mathbf{R}}(\sigma(s))|g(s, c)| \Delta s<\frac{\varepsilon}{4} .
$$

Hence, for $y \in \Omega, t_{2}>t_{1} \geq T^{*}$, we get

$$
\begin{aligned}
\left|(\mathcal{P} y)\left(t_{2}\right)-(\mathcal{P} y)\left(t_{1}\right)\right|= & \mid \int_{T}^{t_{2}} \int_{s_{3}}^{\infty} \frac{1}{r\left(s_{2}\right)} \int_{s_{2}}^{\infty} \int_{s_{1}}^{\infty} g(s, y(\eta(s))) \Delta s \Delta s_{1} \Delta s_{2} \Delta s_{3} \\
& -\int_{T}^{t_{1}} \int_{s_{3}}^{\infty} \frac{1}{r\left(s_{2}\right)} \int_{s_{2}}^{\infty} \int_{s_{1}}^{\infty} g(s, y(\eta(s))) \Delta s \Delta s_{1} \Delta s_{2} \Delta s_{3} \mid \\
\leq & 4 \int_{T^{*}}^{\infty} \widetilde{\mathbf{R}}(\sigma(s))|g(s, y(\eta(s)))| \Delta s \\
\leq & 4 \int_{T^{*}}^{\infty} \widetilde{\mathbf{R}}(\sigma(s))|g(s, c)| \Delta s<\varepsilon,
\end{aligned}
$$

which implies that $\{\mathcal{P} y: y \in \Omega\}$ is uniformly Cauchy.

For any $T_{1} \in \mathbb{T}_{0}$ and $y \in \Omega$, if $T \leq t_{1}<t_{2} \leq T_{1}$, then

$$
\left|(\mathcal{P} y)\left(t_{2}\right)-(\mathcal{P} y)\left(t_{1}\right)\right| \leq\left|\int_{t_{1}}^{t_{2}} \int_{s_{3}}^{\infty} \frac{1}{r\left(s_{2}\right)} \int_{s_{2}}^{\infty} \int_{s_{1}}^{\infty} g(s, y(\eta(s))) \Delta s \Delta s_{1} \Delta s_{2} \Delta s_{3}\right|
$$




$$
\begin{aligned}
\leq & \int_{t_{1}}^{t_{2}} \frac{\sigma\left(s_{2}\right)-t_{1}}{r\left(s_{2}\right)} \int_{s_{2}}^{\infty}\left(\sigma(s)-s_{2}\right)|g(s, y(\eta(s)))| \Delta s \Delta s_{2} \\
& +\int_{t_{2}}^{\infty} \frac{t_{2}-t_{1}}{r\left(s_{2}\right)} \int_{s_{2}}^{\infty}\left(\sigma(s)-s_{2}\right)|g(s, y(\eta(s)))| \Delta s \Delta s_{2} \\
\leq & \left(t_{2}-t_{1}\right)\left[\int_{t_{1}}^{t_{2}} \frac{1}{r\left(s_{2}\right)} \int_{s_{2}}^{\infty}\left(\sigma(s)-s_{2}\right)|g(s, y(\eta(s)))| \Delta s \Delta s_{2}\right. \\
& \left.+\int_{t_{2}}^{\infty} \frac{1}{r\left(s_{2}\right)} \int_{s_{2}}^{\infty}\left(\sigma(s)-s_{2}\right)|g(s, y(\eta(s)))| \Delta s \Delta s_{2}\right] \\
= & \left(t_{2}-t_{1}\right) \int_{t_{1}}^{\infty} \bar{R}_{t_{1}}(\sigma(s))|g(s, y(\eta(s)))| \Delta s,
\end{aligned}
$$

where

$$
\bar{R}_{t_{1}}(t)=\int_{t_{1}}^{t} \frac{t-s}{r(s)} \Delta s=\int_{t_{1}}^{t} \int_{t_{1}}^{\sigma\left(s_{1}\right)} \frac{1}{r(s)} \Delta s \Delta s_{1} .
$$

On the other hand, by L'Hôpital's rule, we get

$$
\lim _{t \rightarrow \infty} \frac{K_{t_{1}}(t)}{\widetilde{\mathbf{K}}_{t_{1}}(t)}=\lim _{t \rightarrow \infty} \frac{1}{\sigma(t)-t_{1}}=0
$$

which, in view of Lemma 3.5, implies that $\lim _{t \rightarrow \infty} \frac{K_{t_{1}}(\sigma(t))}{\widetilde{\mathbf{K}}_{t_{1}}(\sigma(t))}=0$, where

$$
K_{t_{1}}(t)=\int_{t_{1}}^{t} \frac{1}{r(s)} \Delta s \quad \text { and } \quad \widetilde{\mathbf{K}}_{t_{1}}(t)=\int_{t_{1}}^{t} \frac{\sigma(s)-t_{1}}{r(s)} \Delta s .
$$

Furthermore, by using the earlier argument, we find that $\lim _{t \rightarrow \infty} \frac{\bar{R}_{t_{1}}(\sigma(t))}{\widetilde{\mathbf{R}}_{t_{1}}(\sigma(t))}=0$. Hence, for any $\epsilon>0$, there exists $T_{1}^{*} \geq t_{1}$ such that $\bar{R}_{t_{1}}(\sigma(t))<\epsilon \widetilde{\mathbf{R}}_{t_{1}}(\sigma(t))$ for $t \geq T_{1}^{*}$. This means that

$$
\begin{aligned}
\left|(\mathcal{P} y)\left(t_{2}\right)-(\mathcal{P} y)\left(t_{1}\right)\right| \leq & \left(t_{2}-t_{1}\right) \epsilon \int_{T_{1}^{*}}^{\infty} \widetilde{\mathbf{R}}_{t_{1}}(\sigma(s))|g(s, y(\eta(s)))| \Delta s \\
& +\left(t_{2}-t_{1}\right) \int_{t_{1}}^{T_{1}^{*}} \bar{R}_{t_{1}}(\sigma(s))|g(s, y(\eta(s)))| \Delta s .
\end{aligned}
$$

Therefore, there exists $\delta>0$ such that

$$
\left|(\mathcal{P} y)\left(t_{2}\right)-(\mathcal{P} y)\left(t_{1}\right)\right|<\varepsilon, \quad \text { if }\left|t_{2}-t_{1}\right|<\delta .
$$

Moreover, we have

$$
\left|(\mathcal{P} y)\left(t_{2}\right)-(\mathcal{P} y)\left(t_{1}\right)\right|=0<\varepsilon, \quad \text { if } t_{0} \leq t_{1}<t_{2} \leq T .
$$

From the preceding arguments, we conclude that $\{\mathcal{P} y: y \in \Omega\}$ is equi-continuous on $\left[t_{0}, T_{1}\right]$. Hence, $\mathcal{P} \Omega$ is relatively compact. Thus, $\mathcal{P}$ is completely continuous. Hence, by Schauder's fixed point theorem, $\mathcal{P}$ has a fixed point $y_{0} \in \Omega$, which is a nonoscillatory solution of (1). This is a contradiction. 
We next prove the sufficiency by contradiction. Without loss of generality, let $y(t)$ be an eventually positive solution of (1). Then, from Lemma 3.4, there exists $t_{1} \geq t_{0}$ such that, for any $T \geq t_{1}$, we have

$$
y(\sigma(t)) \geq \int_{t_{1}}^{\sigma(t)} \widetilde{\mathbf{R}}_{t_{1}}(\sigma(s)) g(s, y(\eta(s))) \Delta s, \quad t \geq T .
$$

It follows from $y^{\Delta}(t)>0$ for $t \geq t_{1}$ that there exists a constant $c_{1}>0$ such that $y(\eta(t)) \geq c_{1}$ for $t \geq t_{1}$. Then, by the strong superlinearity of $g$, we have

$$
g(t, y(\eta(t))) \geq c_{1}^{-\alpha} y^{\alpha}(\eta(t)) g\left(t, c_{1}\right) \geq c_{1}^{-\alpha} y^{\alpha}(\sigma(t)) g\left(t, c_{1}\right) .
$$

Hence

$$
y(\sigma(t)) \geq c_{1}^{-\alpha} \int_{t_{1}}^{\sigma(t)} \widetilde{\mathbf{R}}_{t_{1}}(\sigma(s)) y^{\alpha}(\sigma(s)) g\left(s, c_{1}\right) \Delta s, \quad t \geq T,
$$

that is,

$$
(y(\sigma(t)))^{-\alpha} \leq c_{1}^{\alpha^{2}}\left(\int_{t_{1}}^{\sigma(t)} \widetilde{\mathbf{R}}_{t_{1}}(\sigma(s)) y^{\alpha}(\sigma(s)) g\left(s, c_{1}\right) \Delta s\right)^{-\alpha}, \quad t \geq T .
$$

Notice that there exists $\zeta \in[s, \sigma(s)]$ such that

$$
\begin{aligned}
& {\left[\left(\int_{t_{1}}^{s} \widetilde{\mathbf{R}}_{t_{1}}(\sigma(\theta)) y^{\alpha}(\sigma(\theta)) g\left(\theta, c_{1}\right) \Delta \theta\right)^{1-\alpha}\right]^{\Delta}} \\
& =(1-\alpha) \widetilde{\mathbf{R}}_{t_{1}}(\sigma(s)) y^{\alpha}(\sigma(s)) g\left(s, c_{1}\right) \\
& \quad \times\left(\int_{t_{1}}^{\zeta} \widetilde{\mathbf{R}}_{t_{1}}(\sigma(\theta)) y^{\alpha}(\sigma(\theta)) g\left(\theta, c_{1}\right) \Delta \theta\right)^{-\alpha} .
\end{aligned}
$$

Multiplying (10) by $\widetilde{\mathbf{R}}_{t_{1}}(\sigma(t)) y^{\alpha}(\sigma(t)) g\left(t, c_{1}\right)$ and then integrating from $t_{2}\left(t_{2}>T\right)$ to $t$, we get

$$
\begin{aligned}
& \int_{t_{2}}^{t} \widetilde{\mathbf{R}}_{t_{1}}(\sigma(s)) g\left(s, c_{1}\right) \Delta s \\
& \quad \leq c_{1}^{\alpha^{2}} \int_{t_{2}}^{t} \widetilde{\mathbf{R}}_{t_{1}}(\sigma(s)) y^{\alpha}(\sigma(s)) g\left(s, c_{1}\right)\left(\int_{t_{1}}^{\zeta} \widetilde{\mathbf{R}}_{t_{1}}(\sigma(\theta)) y^{\alpha}(\theta) g\left(\theta, c_{1}\right) \Delta \theta\right)^{-\alpha} \Delta s \\
& \quad=\left.\frac{c_{1}^{\alpha^{2}}}{(\alpha-1)}\left(\int_{t_{1}}^{s} \widetilde{\mathbf{R}}_{t_{1}}(\sigma(\theta)) y^{\alpha}(\sigma(\theta)) g\left(\theta, c_{1}\right) \Delta \theta\right)^{1-\alpha}\right|_{t} ^{t_{2}} .
\end{aligned}
$$

This means that

$$
\int_{t_{2}}^{t} \widetilde{\mathbf{R}}_{t_{1}}(\sigma(s)) g\left(s, c_{1}\right) \Delta s<\infty .
$$

On the other hand, we have

$$
\widetilde{\mathbf{R}}(t)=\int_{t_{0}}^{t} \frac{(t-s)\left(\sigma(s)-t_{0}\right)}{r(s)} \Delta s=\int_{t_{0}}^{t} \int_{t_{0}}^{\sigma\left(s_{1}\right)} \frac{\sigma(s)-t_{0}}{r(s)} \Delta s \Delta s_{1} .
$$


Now, we show that $\lim _{t \rightarrow \infty} \frac{\widetilde{\mathbf{R}}_{t_{1}}(\sigma(t))}{\widetilde{\mathbf{R}}(\sigma(t))}=1$. In fact, from L'Hôpital's rule and Lemma 3.5, we need to prove that

$$
\lim _{t \rightarrow \infty} \frac{\widetilde{\mathbf{R}}_{t_{1}}(t)}{\widetilde{\mathbf{R}}(t)}=\lim _{t \rightarrow \infty} \frac{\widetilde{\mathbf{R}}_{t_{1}}^{\Delta}(t)}{\widetilde{\mathbf{R}}^{\Delta}(t)}=1
$$

i.e., it is sufficient to show that

$$
\lim _{t \rightarrow \infty} \frac{\widetilde{\mathbf{K}}_{t_{1}}(\sigma(t))}{\widetilde{\mathbf{K}}_{t_{0}}(\sigma(t))}=1 .
$$

Furthermore, one can see that expression (11) is true. Indeed, from L'Hôpital's rule and Lemma 3.5 again, we just need to show that

$$
\lim _{t \rightarrow \infty} \frac{\widetilde{\mathbf{K}}_{t_{1}}(t)}{\widetilde{\mathbf{K}}_{t_{0}}(t)}=\lim _{t \rightarrow \infty} \frac{\widetilde{\mathbf{K}}_{t_{1}}^{\Delta}(t)}{\widetilde{\mathbf{K}}_{t_{0}}^{\Delta}(t)}=1 .
$$

Obviously, (12) is satisfied. Thus, there exists $t_{3} \geq T$ such that $\widetilde{\mathbf{R}}_{t_{1}}(\sigma(t))>\frac{1}{2} \widetilde{\mathbf{R}}(\sigma(t))$ for $t \geq t_{3}$. It means that

$$
\int_{t_{2}}^{\infty} \widetilde{\mathbf{R}}(\sigma(s)) g\left(s, c_{1}\right) \Delta s<\infty
$$

which contradicts (3). The proof is complete.

Theorem 3.7 Assume that $g$ is strongly sublinear and $\eta(t) \leq t$. Then every solution of $(1)$ is oscillatory if and only if

$$
\int_{t_{0}}^{\infty}|g(t, c R(\eta(t)))| \Delta t=\infty \quad \text { for all } c \neq 0 .
$$

Proof We first prove the necessity by contradiction. Suppose that condition (13) does not hold true. Then there exists $c>0$ such that

$$
\int_{t_{0}}^{\infty}|g(t, c R(\eta(t)))| \Delta t<\infty
$$

Let $T>t_{0}$ be so large that

$$
\int_{T}^{\infty}|g(t, c R(\eta(t)))| \Delta t<\frac{c}{2}
$$

Let

$$
U=\left\{y \mid y \in C_{r d}\left(\mathbb{T}_{0}, \mathbb{R}\right), \sup _{t \in \mathbb{T}_{0}} \frac{|y(t)|}{R^{2}(t)}<\infty\right\}
$$

Obviously, $U$ is a Banach space with the norm $\|y\|=\sup _{t \in \mathbb{T}_{0}} \frac{|y(t)|}{R^{2}(t)}$. Define a closed and convex subset of $U$ as follows:

$$
\Omega=\left\{y=y(t): y \in U, c R(t) \leq|y(t)| \leq 2 c R(t), t \in \mathbb{T}_{0}\right\} .
$$


We define a map $\mathcal{P}$ on $\Omega$ by

$$
(\mathcal{P} y)(t)= \begin{cases}c R(t)+\int_{T}^{t} \int_{T}^{s_{3}} \frac{1}{r\left(s_{2}\right)} \int_{T}^{s_{2}} \int_{s_{1}}^{\infty} g(s, y(\eta(s))) \Delta s \Delta s_{1} \Delta s_{2} \Delta s_{3}, & t \geq T, \\ (\mathcal{P} y)(T), & t_{0} \leq t \leq T .\end{cases}
$$

In order to show that $\mathcal{P}$ has a fixed point in $\Omega$, we proceed as follows.

Step I. P maps $\Omega$ into $\Omega$. Let $y \in \Omega$. Then $c R(t) \leq|y(t)| \leq 2 c R(t)$ for $t \geq t_{0}$. Furthermore, we have

$$
\begin{aligned}
|(\mathcal{P} y)(t)| \leq & c R(t)+\int_{T}^{t} \int_{T}^{s_{3}} \frac{1}{r\left(s_{2}\right)}\left[\int_{T}^{s_{2}}(\sigma(s)-T)|g(s, y(\eta(s)))| \Delta s\right. \\
& \left.+\int_{s_{2}}^{\infty}\left(s_{2}-T\right)|g(s, y(\eta(s)))| \Delta s\right] \Delta s_{2} \Delta s_{3} \\
\leq & c R(t)+\int_{T}^{t} \int_{T}^{s_{3}} \frac{s_{2}-T}{r\left(s_{2}\right)}\left[\frac{c}{2}+\frac{c}{2}\right] \Delta s_{2} \Delta s_{3} \\
\leq & c R(t)+c R(t)=2 c R(t),
\end{aligned}
$$

which implies that $c R(t) \leq|(\mathcal{P} y)(t)| \leq 2 c R(t)$ for $t \in \mathbb{T}_{0}$. This shows that $\mathcal{P} \Omega \subseteq \Omega$.

Step II. $\mathcal{P}$ is completely continuous.

Firstly, we show that $\mathcal{P}$ is continuous. Set $y_{n} \in \Omega$ and $\left\|y_{n}-y\right\| \rightarrow 0$ as $n \rightarrow \infty$. Hence, we have $y \in \Omega$ since $\Omega$ is a closed set. Then

$$
\begin{aligned}
&\left|\left(\mathcal{P} y_{n}\right)(t)-(\mathcal{P} y)(t)\right| \\
& \leq \int_{T}^{t} \int_{T}^{s_{3}} \frac{1}{r\left(s_{2}\right)} \int_{T}^{s_{2}} \int_{s_{1}}^{\infty}\left|g\left(s, y_{n}(\eta(s))\right)-g(s, y(\eta(s)))\right| \Delta s \Delta s_{1} \Delta s_{2} \Delta s_{3} \\
& \leq \int_{T}^{t} \int_{T}^{s_{3}} \frac{1}{r\left(s_{2}\right)}\left[\int_{T}^{s_{2}}(\sigma(s)-T)\left|g\left(s, y_{n}(\eta(s))\right)-g(s, y(\eta(s)))\right| \Delta s\right. \\
&\left.\quad+\int_{s_{2}}^{\infty}\left(s_{2}-T\right)\left|g\left(s, y_{n}(\eta(s))\right)-g(s, y(\eta(s)))\right| \Delta s\right] \Delta s_{2} \Delta s_{3} \\
& \leq \int_{T}^{t} \int_{T}^{s_{3}} \frac{\left(s_{2}-T\right)}{r\left(s_{2}\right)} \Delta s_{2} \Delta s_{3} \int_{T}^{\infty}\left|g\left(s, y_{n}(\eta(s))\right)-g(s, y(\eta(s)))\right| \Delta s \\
& \leq \int_{T}^{\infty}\left|g\left(s, y_{n}(\eta(s))\right)-g(s, y(\eta(s)))\right| \Delta s \times R(t) .
\end{aligned}
$$

By the strong sublinearity of $g$, we have

$$
\begin{aligned}
& |g(s, y(\eta(s)))| \leq 2^{\alpha} \mid g(s, c R((\eta(s))) \mid, \quad \text { and } \\
& \left|g\left(s, y_{n}(\eta(s))\right)\right| \leq 2^{\alpha}|g(s, c R(\eta(s)))|, \quad n=1,2 \ldots
\end{aligned}
$$

Then

$$
\left|g\left(s, y_{n}(\eta(s))\right)-g(s, y(\eta(s)))\right| \leq 2^{\alpha+1}|g(s, c R((\eta(s))))| .
$$

Since $\left|g\left(s, y_{n}(\eta(s))\right)-g(s, y(\eta(s)))\right| \rightarrow 0$ as $n \rightarrow \infty$, the Lebesgue dominated convergence theorem implies that $\lim _{n \rightarrow \infty}\left\|\mathcal{P} y_{n}-\mathcal{P} y\right\|=0$, and thus $\mathcal{P}$ is continuous in $\Omega$. 
We next show that $\mathcal{P} \Omega$ is relatively compact. The boundedness is obvious. For any $\varepsilon>0$, let $T^{*} \geq T$ be so large that

$$
\left|\frac{1}{R(t)}\right|<\frac{\varepsilon}{4 c} \quad \text { for } t \geq T^{*} .
$$

Hence, for $y \in \Omega, t_{2}>t_{1} \geq T^{*}$,

$$
\begin{aligned}
&\left|\left(R^{-2} \mathcal{P} y\right)\left(t_{2}\right)-\left(R^{-2} \mathcal{P} y\right)\left(t_{1}\right)\right| \\
& \leq \frac{c}{R\left(t_{2}\right)}+\frac{1}{R^{2}\left(t_{2}\right)} \int_{T}^{t_{2}} \int_{T}^{s_{3}} \frac{1}{r\left(s_{2}\right)} \int_{T}^{s_{2}} \int_{s_{1}}^{\infty}|g(s, y(\eta(s)))| \Delta s \Delta s_{1} \Delta s_{2} \Delta s_{3} \\
& \quad+\frac{c}{R\left(t_{1}\right)}+\frac{1}{R^{2}\left(t_{1}\right)} \int_{T}^{t_{1}} \int_{T}^{s_{3}} \frac{1}{r\left(s_{2}\right)} \int_{T}^{s_{2}} \int_{s_{1}}^{\infty}|g(s, y(\eta(s)))| \Delta s \Delta s_{1} \Delta s_{2} \Delta s_{3} \\
& \leq \frac{c}{R\left(t_{2}\right)}+\frac{c}{R\left(t_{1}\right)}+\frac{c}{R^{2}\left(t_{2}\right)} \int_{T}^{t_{2}} \int_{T}^{s_{3}} \frac{s_{2}-T}{r\left(s_{2}\right)} \Delta s_{2} \Delta s_{3}+\frac{c}{R^{2}\left(t_{1}\right)} \int_{T}^{t_{1}} \int_{T}^{s_{3}} \frac{s_{2}-T}{r\left(s_{2}\right)} \Delta s_{2} \Delta s_{3} \\
& \leq \frac{c}{R\left(t_{2}\right)}+\frac{c}{R\left(t_{1}\right)}+\frac{c}{R\left(t_{2}\right)}+\frac{c}{R\left(t_{1}\right)}<\varepsilon
\end{aligned}
$$

which implies that $\{\mathcal{P} y: y \in \Omega\}$ is uniformly Cauchy. Furthermore, for any $y \in \Omega$ and $T_{1} \in \mathbb{T}_{0}$, if $T \leq t_{1}<t_{2} \leq T_{1}$, then

$$
\begin{aligned}
\left|\left(R^{-2} \mathcal{P} y\right)\left(t_{2}\right)-\left(R^{-2} \mathcal{P} y\right)\left(t_{1}\right)\right| \\
\leq\left|\frac{c}{R\left(t_{2}\right)}-\frac{c}{R\left(t_{1}\right)}\right| \\
\quad+\mid \frac{1}{R^{2}\left(t_{2}\right)} \int_{T}^{t_{2}} \int_{T}^{s_{3}} \frac{1}{r\left(s_{2}\right)} \int_{T}^{s_{2}} \int_{s_{1}}^{\infty} g(s, y(\eta(s))) \Delta s \Delta s_{1} \Delta s_{2} \Delta s_{3} \\
\quad-\frac{1}{R^{2}\left(t_{1}\right)} \int_{T}^{t_{1}} \int_{T}^{s_{3}} \frac{1}{r\left(s_{2}\right)} \int_{T}^{s_{2}} \int_{s_{1}}^{\infty} g(s, y(\eta(s))) \Delta s \Delta s_{1} \Delta s_{2} \Delta s_{3} \mid \\
\leq\left|\frac{c}{R\left(t_{2}\right)}-\frac{c}{R\left(t_{1}\right)}\right| \\
\quad+\left|\frac{1}{R^{2}\left(t_{2}\right)}-\frac{1}{R^{2}\left(t_{1}\right)}\right| \int_{T}^{t_{2}} \int_{T}^{s_{3}} \frac{1}{r\left(s_{2}\right)} \int_{T}^{s_{2}} \int_{s_{1}}^{\infty}|g(s, y(\eta(s)))| \Delta s \Delta s_{1} \Delta s_{2} \Delta s_{3} \\
\quad+\frac{1}{R^{2}\left(t_{1}\right)} \int_{t_{1}}^{t_{2}} \int_{T}^{s_{3}} \frac{1}{r\left(s_{2}\right)} \int_{T}^{s_{2}} \int_{s_{1}}^{\infty}|g(s, y(\eta(s)))| \Delta s \Delta s_{1} \Delta s_{2} \Delta s_{3} \\
\leq\left|\frac{c}{R\left(t_{2}\right)}-\frac{c}{R\left(t_{1}\right)}\right|+\left|\frac{1}{R^{2}\left(t_{2}\right)}-\frac{1}{R^{2}\left(t_{1}\right)}\right| \times c R\left(t_{2}\right) \\
\quad+\frac{c}{R^{2}\left(t_{1}\right)} \int_{t_{1}}^{t_{2}} \int_{T}^{s_{3}} \frac{s_{2}}{r\left(s_{2}\right)} \Delta s_{2} \Delta s_{3} .
\end{aligned}
$$

Hence, there exists $\delta>0$ such that

$$
\left|\left(R^{-2} \mathcal{P} y\right)\left(t_{2}\right)-\left(R^{-2} \mathcal{P} y\right)\left(t_{1}\right)\right|<\varepsilon, \quad \text { if }\left|t_{2}-t_{1}\right|<\delta .
$$

Moreover, we have

$$
\left|\left(R^{-2} \mathcal{P} y\right)\left(t_{2}\right)-\left(R^{-2} \mathcal{P} y\right)\left(t_{1}\right)\right|=0<\varepsilon, \quad \text { if } t_{0} \leq t_{1}<t_{2} \leq T .
$$


In consequence, $\{\mathcal{P} y: y \in \Omega\}$ is equi-continuous on $\left[t_{0}, T_{1}\right]$. According to the ArzelaAscoli theorem on time scales, we know that $\mathcal{P}$ is a compact operator. Hence $\mathcal{P}$ is completely continuous. Therefore, $\mathcal{P}$ has a fixed point $y_{0} \in \Omega$ according to Schauder's fixed point theorem, which is a nonoscillatory solution of (1). This is a contradiction.

Now, we prove the sufficiency by contradiction. Without loss of generality, let $y(t)$ be an eventually positive solution of (1). From Lemmas 3.1 and 3.3, there exist $t_{1} \geq t_{0}$ and a positive constant $c_{1}$ such that

$$
y(t)>0, \quad y^{\Delta}(t)>0, \quad \text { and } \quad\left[r(t) y^{\Delta \Delta}(t)\right]^{\Delta}>0, \quad t \geq t_{1},
$$

and

$$
\frac{1}{2}\left[r(t) y^{\Delta \Delta}(t)\right]^{\Delta} R(t) \leq y(t) \leq c_{1} R(t), \quad t \geq t_{1} .
$$

Noting that $\left[r(t) y^{\Delta \Delta}(t)\right]^{\Delta \Delta}<0$, we have

$$
y(\eta(t)) \geq\left.\frac{1}{2}\left[r(s) y^{\Delta \Delta}(s)\right]^{\Delta}\right|_{s=\eta(t)} R(\eta(t)) \geq \frac{1}{2}\left[r(t) y^{\Delta \Delta}(t)\right]^{\Delta} R(\eta(t)) .
$$

From (14), (15), and the strong sublinearity of $g$, there exists $\zeta \in[t, \sigma(t)]$ such that

$$
\begin{aligned}
\left(-\left(\left[r(t) y^{\Delta \Delta}(t)\right]^{\Delta}\right)^{1-\alpha}\right)^{\Delta} & =-(1-\alpha)\left(\left[r(\zeta) y^{\Delta \Delta}(\zeta)\right]^{\Delta}\right)^{-\alpha}\left[r(\zeta) y^{\Delta \Delta}(\zeta)\right]^{\Delta \Delta} \\
& =(1-\alpha)\left(\left[r(\zeta) y^{\Delta \Delta}(\zeta)\right]^{\Delta}\right)^{-\alpha} g(t, y(\eta(t))) \\
& \geq(1-\alpha)\left(\left[r(t) y^{\Delta \Delta}(t)\right]^{\Delta}\right)^{-\alpha} \frac{(y(\eta(t)))^{\alpha}}{\left(c_{1} R(\eta(t))\right)^{\alpha}} g\left(t, c_{1} R(\eta(t))\right) \\
& \geq(1-\alpha) \frac{1}{\left(2 c_{1}\right)^{\alpha}} g\left(t, c_{1} R(\eta(t))\right) .
\end{aligned}
$$

Integrating the inequalities above from $t_{2}$ to $t$, we get

$$
(1-\alpha) \frac{1}{\left(2 c_{1}\right)^{\alpha}} \int_{t_{2}}^{t} g\left(s, c_{1} R(\eta(s))\right) \Delta s \leq\left(\left[r\left(t_{2}\right) y^{\Delta \Delta}\left(t_{2}\right)\right]^{\Delta}\right)^{1-\alpha}-\left(\left[r(t) y^{\Delta \Delta}(t)\right]^{\Delta}\right)^{1-\alpha}<\infty
$$

and so

$$
\int_{t_{2}}^{\infty} g\left(s, c_{1} R(\eta(s))\right) \Delta s<\infty
$$

which contradicts (13). This completes the proof.

Remark 3.8 It is noteworthy that the results given in the aforementioned theorems are the same as those in [13] where the dynamic equation on time scales is reduced to a differential equation when $\mathbb{T}=\mathbb{R}_{+}, \sigma(t)=t$, and $x^{\Delta}=x^{\prime}$. If further we set $r(t)=1$ and $\eta(t)=\sin t$ in (1), we conclude that $R(t)=\widetilde{\mathbf{R}}(t)=t^{3} / 6$, and every solution of (1) is oscillatory in view of Theorem 3.7 whenever (13) holds under a suitable strongly sublinear function $g$. However, for the case of $\mathbb{T}=\mathbb{N}$, we know that $\sigma(t)=t+1$ and $x^{\Delta}(t)=\Delta x(t)=x(t+1)-x(t)$, there is no 
work concerning the sufficient and necessary conditions for the following corresponding difference equation:

$$
\Delta^{2}\left[r(t) \Delta^{2} x(t)\right]+g(t, x(\eta(t)))=0 .
$$

Example 3.9 Let $\mathbb{T}=\{t: t \in \mathbb{N}\}, r(t)=\frac{t+1}{2 t}, \eta(t)=\lambda t$ for $\lambda>2$, and $g(t, x(t))=x^{3}(t)$ in (16). It is easy to see that $\sum_{t=1}^{\infty} t / r(t)=\infty$ and $\sum_{t=1}^{\infty} \widetilde{\mathbf{R}}(t+1)\left|c^{3}\right|=\infty$ for $c \neq 0$, and then all the conditions of Theorem 3.6 are satisfied. Thus every solution of (16) is oscillatory.

Example 3.10 Consider the fourth order dynamic equation

$$
\left[t y^{\Delta \Delta}(t)\right]^{\Delta \Delta}+\frac{1}{t} y^{5}(4 t)=0, \quad t \in 2^{\mathbb{N}}, t \geq t_{0}=2
$$

Here, $\mathbb{T}=2^{\mathbb{N}}, \eta(t)=4 t$ and $g(t, y(t))=\frac{1}{t} y^{5}(t)$. Hence we have $\sigma(t)=2 t$. In this case $r(t)=t$, one can check that $\int_{2}^{\infty} t / r(t) \Delta t=\infty$ and $\widetilde{\mathbf{R}}(t)=\frac{4}{3} t^{2}-\frac{4}{3}-2 t \frac{\ln t}{\ln 2}$. Hence $\int_{2}^{\infty} \widetilde{\mathbf{R}}(2 t)|g(t, c)| \Delta t=$ $\infty$. It means that all the conditions of Theorem 3.6 are satisfied. Then every solution of (17) is oscillatory.

\section{Funding}

The work was supported by the National Natural Science Foundation of China (11671339).

\section{Availability of data and materials}

Not applicable.

\section{Competing interests}

The authors declare that they have no competing interests.

\section{Authors' contributions}

Each of the authors, $\mathrm{YZ}$, JWH, BA, and AA, contributed equally to each part of this work. All authors read and approved the final manuscript.

\section{Publisher's Note}

Springer Nature remains neutral with regard to jurisdictional claims in published maps and institutional affiliations.

Received: 6 May 2019 Accepted: 16 July 2019 Published online: 30 July 2019

\section{References}

1. Bohner, M., Peterson, A.: Dynamic Equations on Time Scales: An Introduction with Applications. Birkhäuser, Boston (2001)

2. Bohner, M., Georgiev, S.G.: Multivariable Dynamic Calculus on Time Scales. Springer, Berlin (2017)

3. Georgiev, S.G.: Fractional Dynamic Calculus and Fractional Dynamic Equations on Time Scales. Springer, Berlin (2018)

4. Martynyuk, A.A.: Stability Theory for Dynamic Equations on Time Scales. Springer, Berlin (2016)

5. Došlý, O., Hilger, S.: A necessary and sufficient condition for oscillation of the Sturm-Liouville dynamic equation on time scales. J. Comput. Appl. Math. 141, 147-158 (2002)

6. Zhu, Z.Q., Wang, Q.R.: Existence of nonoscillatory solutions to neutral dynamic equations on time scales. J. Math. Anal. Appl. 335(2), 751-762 (2007)

7. Karpuz, B:: Necessary and sufficient conditions on the asymptotic behavior of second-order neutral delay dynamic equations with positive and negative coefficients. Math. Methods Appl. Sci. 37(8), 1219-1231 (2014)

8. Zhou, Y.: Nonoscillation of higher order neutral dynamic equations on time scales. Appl. Math. Lett. 94, 204-209 (2019)

9. Zhou, Y., Ahmad, B., Alsaedi, A.: Necessary and sufficient conditions for oscillation of second-order dynamic equations on time scales. Math. Methods Appl. Sci. (2019) https://doi.org/10.1002/mma.5672

10. Bohner, M., Hassan, T.S., Li, T.: Fite-Hille-Wintner-type oscillation criteria for second order half-linear dynamic equations with deviating arguments. Indag. Math. 29, 548-560 (2018)

11. Onose, H.: Nonlinear oscillation of fourth order functional differential equations. Ann. Mat. Pura Appl. 119, 259-272 (1979)

12. Kusano, T., Naito, M.: Nonlinear oscillation of fourth order differential equations. Can. J. Math. 28, 840-852 (1976)

13. Gopalsamy, K., Wen, L., Chen, Y., et al.: Necessary and sufficient conditions for a fourth order functional differential equation to be oscillatory. Math. Nachr. 164, 23-36 (1993) 
14. Zhang, C., Li, T., Agarwal, R.P., et al.: Oscillation results for fourth-order nonlinear dynamic equations. Appl. Math. Lett. 25, 2058-2065 (2012)

15. Qi, Y., Yu, J.: Oscillation criteria for fourth-order nonlinear delay dynamic equations. Electron. J. Differ. Equ. 2013, 79, 1314-1344 (2013)

16. Agarwal, R.P., Bohner, M., Li, T., et al.: Oscillation theorems for fourth-order half-linear delay dynamic equations with damping. Mediterr. J. Math. 11, 463-475 (2014)

17. Grace, S.R., Agarwal, R.P., Saejie, W.: Monotone and oscillatory behavior of certain fourth order nonlinear dynamic equations. Dyn. Syst. Appl. 19, 25-32 (2010)

18. Grace, S.R., Sun, S., Wang, Y.: On the oscillation of fourth order strongly superlinear and strongly sublinear dynamic equations. J. Appl. Math. Comput. 44(1-2), 119-132 (2014)

19. Li, T., Thandapani, E., Tang, S.: Oscillation theorems for fourth-order delay dynamic equations on time scales. Bull. Math. Anal. Appl. 3(3), 190-199 (2011)

20. Li, T., Zhang, C., Thandapani, E.: Asymptotic behavior of fourth-order neutral dynamic equations with noncanonical operators. Taiwan. J. Math. 18, 1003-1019 (2014)

21. Zhang, C., Agarwal, R.P., Bohner, M., et al.: Oscillation of fourth-order delay dynamic equations. Sci. China Math. 58 143-160 (2015)

\section{Submit your manuscript to a SpringerOpen ${ }^{\circ}$} journal and benefit from:

- Convenient online submission

- Rigorous peer review

- Open access: articles freely available online

- High visibility within the field

- Retaining the copyright to your article

Submit your next manuscript at $\gg$ springeropen.com 